\title{
2D and 3D Representations for Feature Recognition in Time Geographical Diary Data
}

\author{
Katerina Vrotsou, Camilla Forsell and Matthew Cooper
}

\section{Linköping University Post Print}

\section{Tweet}

N.B.: When citing this work, cite the original article.

Original Publication:

Katerina Vrotsou, Camilla Forsell and Matthew Cooper, 2D and 3D Representations for Feature Recognition in Time Geographical Diary Data, 2010, Information Visualization, (9), 4, 263 276.

http://dx.doi.org/10.1057/ivs.2009.30

Copyright: Palgrave Macmillan / SAGE Publications (UK and US) http://www.uk.sagepub.com/home.nav

Postprint available at: Linköping University Electronic Press

http://urn.kb.se/resolve?urn=urn:nbn:se:liu:diva-52360 


\title{
2D and 3D Representations for Feature Recognition in Time Geographical Diary Data
}

\author{
Katerina Vrotsou, Camilla Forsell, Matthew Cooper \\ Norrköping Visualization and Interaction Studio (NVIS) \\ Linköping University, Sweden
}

\begin{abstract}
Time geographical representations are becoming a common approach to analysing spatio-temporal data. Such representations appear intuitive in the process of identifying patterns and features since paths of populations form tracks through the 3D space which can be seen converging and diverging over time. In this paper we compare 2D and 3D representations within a time geographical visual analysis tool for activity diary data. We define a set of representative tasks and evaluate task performance between the two representations. The results show that the 3D representation has benefits over the $2 \mathrm{D}$ representation for feature identification but also indicate that these benefits can be lost if the 3D representation is not carefully constructed to permit the user to see them.
\end{abstract}

Keywords: Evaluation, 2D and 3D representation, time geography, time diary data.

\section{Introduction}

Human activities and the effect they have on society and nature are of increasing concern and there is an urgent need to gain insights into causes and effects in the behaviour of populations as well as individuals or subgroups of the population. The data describing the populations is intrinsically coupled to geo-temporal information and development of effective methods that support the analysis of three-dimensional (3D) representations of time-based population data is thus a topic of highest relevance. Such methods frequently employ the time geographical cube as originally described by Torsten Hägerstrand [1] in 1970 and re-implemented in 2004 by Kapler et.al [2]. In each case the geographical element is represented by a position in a 2D 'map' plane and the time element by the vertical dimension above the flat map. This allows an individual to be observed moving through the space, and enables identification of when events occur and observation of the subjects interactions and collective actions, while maintaining a sense of the subjects as individuals. The time-cube has also been used in a different form where the geographical element is replaced with an abstract 'activity/individual' space [3] which allows the detailed study of the individuals' activities throughout the time-period, and the identification of patterns of activities [4] in populations. 3D representations have been assumed to be the most intuitive to use as time can be considered an extra dimension over the, usually, two dimensional (2D) space. They are also believed to provide an easy way to examine such data and compare the complex paths of a number of individuals simultaneously. Our experiences in this area lie within the social science field and the use of an activity/individual space for the exploration of daily life. We have found, however, that our users do not fully utilize the potential of the 3D representation in the manner we had anticipated. The goal of this paper is to determine if this is due to a fundamental limitation of the 3D representation, which makes it less useful than expected, or whether the problem lies in some other area such as simply a lack of training or familiarity with 3D representations.

The use of 3D time cube representations relies on the user being able to identify similarities in tracks through the 3D space, whether geographically based or abstract, and requires the user to locate regions where tracks have similar characteristics such as similar transitions, proximity in space or space-time, or simply events occurring at the same time. This paper tests the ability of the user to 
identify such features in a 3D time cube and to determine the relative effectiveness of such a system with respect to an alternative interactive $2 \mathrm{D}$ representation. To achieve this, a set of representative tasks has been identified and controlled experiments carried out within a time-geographical diary data set obtained from the field of social sciences. The results will be indicative of the general effectiveness of the 3D time cube approach and will help to determine factors affecting it.

The major contributions of this paper are:

- The identification of representative tasks in time geographical environments.

- Controlled user-based evaluations to determine the relative effectiveness and efficiency of $2 \mathrm{D}$ and 3D methods in such environments.

- The identification of a number of improvements and potential pitfalls for time geographical displays, helping to make them as effective and efficient as possible.

The remainder of this paper is structured as follows. Section 2 presents some relevant background and related work. The following section provides a general description of our visualization application VISUAL-TimePAcTS. Section 4 describes the experiments performed. Finally, section 5 discusses the general results, proposes new features for the tool and outlines some issues for future work.

\section{Related Work}

Time geography is a geographical conceptual framework [1] in which populations are considered as groups of socially and geographically interrelated individuals and not as indivisible masses. Each individual is unique and their actions are defined and constrained by their location in time and space, by socioeconomic rules and conventions, and by past experiences and knowledge. Time is a continuously changing variable that constrains the individuals' actions in the future, since an individual can be at only one place at a time and can perform a limited range of activities at each time point [5]. An individual's movement in space and time can, therefore, be represented by a single continuous trajectory called a 'space-time path', as seen in Figure 1. Drawing several individuals' space-time paths in the same scene can reveal when, where and for how long paths meet, forming so called 'bundles' [1] (Figure 1). These meetings of paths can be preplanned, such as meetings among friends, family members or colleagues; or coincidental, for example people shopping at the same store, taking the same bus or going to the bank at the same time. The fact that, in most cases, individuals in a society do not collectively plan their days makes the study of 'bundles' interesting with respect to the information they convey about how a society is structured.

According to Peuquet "the manner in which data are represented is inextricably linked with specific analytic tasks" [6]. In order, therefore, to be able to answer questions concerning people in space and time the three components that need to be taken into consideration are: locations (where), times (when), and objects (in our case activities) (what) [6]. Furthermore, when the focus lies with people (human spatio-temporal behaviour), the path/journey itself is not the most significant issue, it is what is happening at the destination (when not moving) that matters $[7,8]$. The where and when are then often the means to understanding the what. The intuitive ability of time geography to reveal spatiotemporal relationships within and between individuals' space-time paths is thus advantageous in the study of human spatio-temporal behaviour. There are, however, many studies of the importance of what people do when 'stationary' that do not use time geographical representations. Forer, in the presentation of his discrete space-time model [9], considers the focus of studying urban interaction to be on individual accessibility and opportunity, and defines accessibility as the ability of actors to meet for at least the minimum time required for their purpose. Dykes and Mountain [10] talk about how 'episodes' of spatio-temporal behaviour defining individuals' activities can be identified by studying the 'breaks' in their positioning tracks and give examples of how this can be done through a visualization process combining spatial and temporal displays with interactive techniques. Kwan [11] 


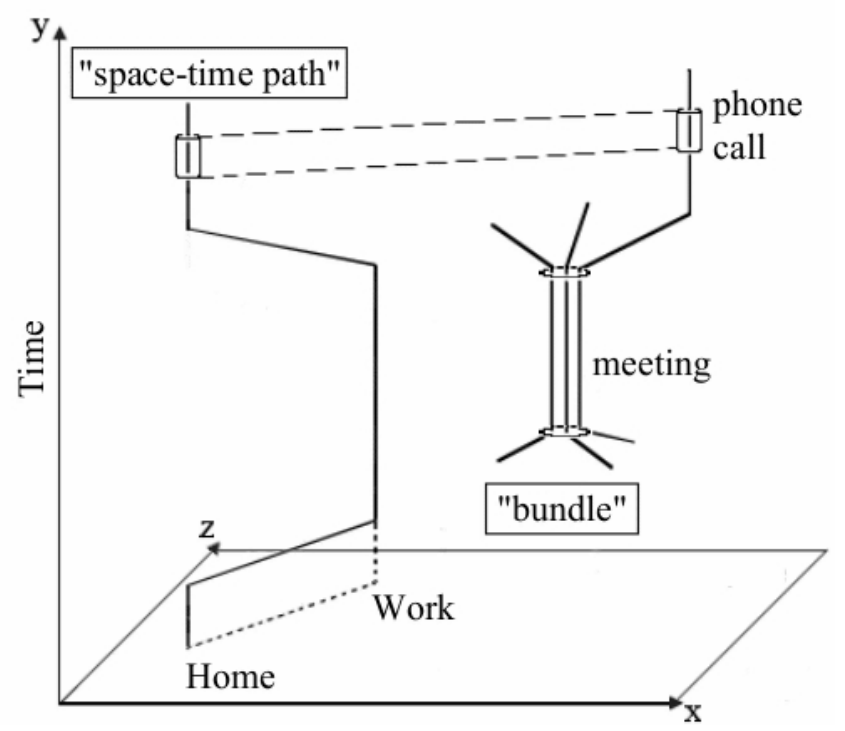

Figure 1: Examples of the traditional time geographical representation as described by Hägerstrand [1]. To the left there is a simple space-time path starting from home going to work and returning to home. To the right several space-time paths meet, forming a bundle in space and time. The telephone call shown in the image is a bundle in time but not in space.

uses 'activity duration lines' and 'activity density surfaces' to reveal human activity patterns in the Portland metropolitan area. Zhao et.al. [12], using time use data collected in the Halifax metropolitan area, combine several visual representations, such as maps, activity rods and ringmaps, in order to reveal patterns of the population's daily activities.

Miller [13] points out that even though geographic information system (GIS) platforms are well suited for place-based studies there is a need for extensions that apply to people-based studies. The time geographical framework provides such an extension but must be expanded further, with the help of information technologies and developments in GIS, in order to handle the growing quality and quantity of data. Many studies have been performed in this area. Kraak [14] implemented the traditional spacetime cube in an interactive visualization environment. A GIS data model is presented by $\mathrm{Yu}$ [15] for analysing spatial and temporal patterns and interactions of human activities. In this work the time geographical concepts were enhanced to better deal with relationships between individuals by defining four new concepts concerned with human interactions. A 'synchronous presence' where paths coincide in both space and time, an 'asynchronous presence' meaning coincidence only in time, a 'synchronous telepresence' where paths coincide only in time, and an 'asynchronous telepresence' where paths do not coincide. Kwan in a substantial body of work, for example [16, 11, 17], has used time geographical representations (path and cube/aquarium) in combination with GIS to reveal human activity patterns, this work is summarized in Geografiska Annaler [18] in 2004. According to Kwan [17], among the disadvantages of using 3D methods is the orientation of the user in the scene, the many visual possibilities and the visual combination effects, meaning problems in identifying relations when many layers or variables are viewed simultaneously, thus leading to clutter.

There are also examples of how other kinds of spatio-temporal event data, which are not human related, can be analysed using time geographical representations. Galatsky et.al [19], for example, use the time cube for visualizing unconnected earthquake events while Turdukulov et.al [20] use a time cube-like representation, the event graph, for showing events in the paths of clouds in time and space. These representations tend to become overwhelming with a large data size due to clutter. Brushing, time windows and using additional linked views displaying complementary information are examples of ways to improve them.

In this related work we have presented the importance of the 'human element' in human geospatial 
behaviour which can be addressed by considering the breaks or discrete events in individuals' paths through time and we have introduced several studies concerned with this. We have further discussed that the interactive 3D space-time cube has, in recent years, made it's way back into the research of time varying data because of the belief in it's intuitive ability to reveal relationships in 3D space-time. As has been noted, however, a 3D representation, such as the time cube, can be problematic due to visual cluttering and problems of orientation of the user relative to the data. Despite these facts, very little work has been done to prove that the 3D time cube, in it's present interactive form, is in fact suitable for visual exploration and identification of patterns within this type of data. To our knowledge the only attempt to evaluate the usefulness of the space-time cube was made by Kristensson et.al [21], where a 3D space-time cube was compared with a 2D map with text markup for the task of analysing four students' walking paths in a university campus area. The results of this study showed no significant difference between the two representations with respect to both errors and response time. The questions asked in the performed experiments, however, are too precise and rather simple which does not make them appropriate to represent a data exploration procedure since the users are not required to explore the whole data set. Furthermore, the 2D map representation is quite cluttered by the text markups despite the small size of the data set. In fact, the behaviour of the representations with respect to an increase in the number of studied individuals is not addressed. These are issues that are important in drawing conclusions about the usefulness of such a 3D representation.

Consequently, the goal of the work presented in this paper is to compare 2D and 3D time geographical representations for feature identification in an exploratory data analysis process, and to determine whether and how performance can be aided by them.

\section{$3 \quad$ Visual Analysis of Activity Diary Data}

The original time geographical concept of the 'space-time path', as seen in the related work (section 2), accounts mainly for an individual's spatial movement over time while the activities performed by the individual (if considered) are implicitly derived from the places visited during this space-time movement [5]. When the objective is to draw conclusions about interaction and human activity patterns, however, the focus should be on the activities performed at the stationary points of a path [7]. These activities are similar to the movements in that they too occur sequentially, each completing before the next can start, they have a location in time and a duration. Therefore, the original time geographical concept has been extended to also consider performed activities [3]. The individuals' daily activity sequences are here too, as in the original time geographical framework, represented by a single continuous trajectory, called an 'activity path' (Figure 2). Similar to the study of space-time paths, many activity paths can be studied simultaneously thus revealing similarities in populations' daily behaviours. This can give information about the way people coexist and can be interesting from many respects. In a local household level to study the daily rhythm of family life: who does what and when, and who helps who; and also at a more global level for studying how communities are structured: when do people perform certain activities such as shopping for food, travelling by bus or going to the bank; how many perform these at the same time and for how long do they engage in them? Comparing these patterns with the opening hours and schedules of the different public services, for example, can lead to an optimization of this community structure.

This extension of the time geographical framework from a geographical space to an abstract activity space [3] is the representation used in this research. This representation was combined with computer graphics, a database and interactive visualization techniques in a visual analysis tool, called VISUAL-TimePAcTS, used for interactively exploring everyday life $[22,23,24,4]$. This section describes the activity diary data used for this study and the visual representations of these activity diaries which will be evaluated within VISUAL-TimePAcTS. 


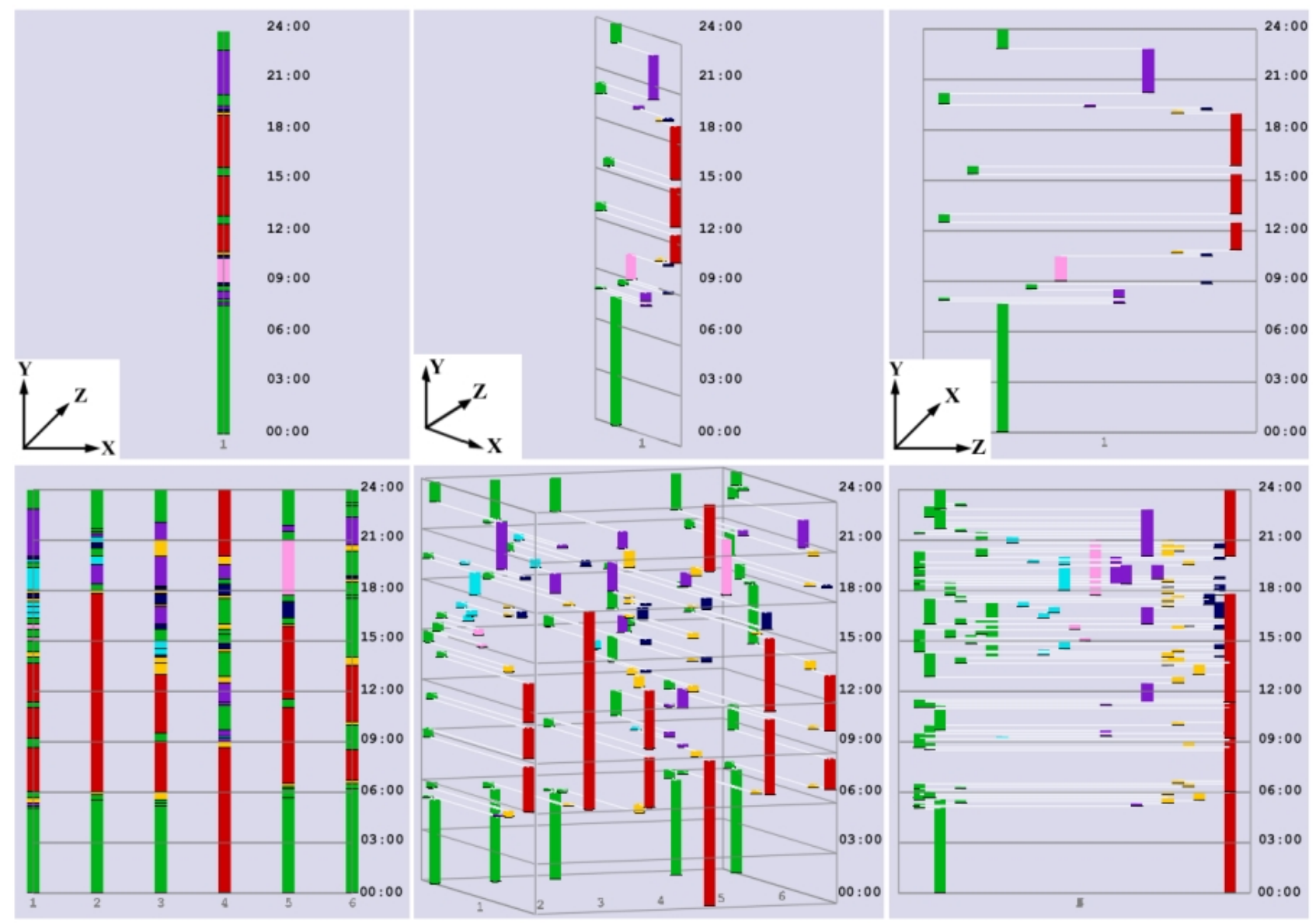

Figure 2: Visual representation of a single activity path and an activity-time cube of 6 activity paths in VISUAL-TimePAcTS. From left to right: front, rotated and side view. Time is represented in the $\mathrm{y}$-axis, individuals on the $\mathrm{x}$-axis and activities on the $\mathrm{z}$-axis. Colour is used to describe the activities at the most general LOD: (1) care for oneself (green), (2) care for others (turqoise), (3) household care (pink), (4) reflection/recreation (purple), (5) transportation (yellow), (6) procure and prepare food (blue), and (7) work/school (red).

\subsection{Activity Diary Data}

The visual analysis tool VISUAL-TimePAcTS has been used with numerous data sets by different user groups. The data set from which we have drawn our test data for this work, consists of coded handwritten activity time diaries collected by the Swedish national statistics bureau (SCB) in 1996. Activity time diaries are frequently used in time-use research for studying populations' daily habits and routines and drawing more detailed conclusions about how people spend their time on a daily basis. Different representations and analysis methods are used for doing so.

The data that we have used include 462 individuals in 179 Swedish households of differing characteristics (singles, couples, with and without children etc.) and in different types of regions (rural and urban). The individuals logged their performed activities, locations and companionship during one week day and one weekend day. A diary entry was made at the start of every new activity. The logged activity data was then categorized into a predefined hierarchical set of some 600 numerical codes, with 5 levels of detail (LOD) with respect to the description of the activity, and grouped under seven general categories, called 'activity spheres' (which also constitute the most general LOD): (1) care for oneself, (2) care for others, (3) household care, (4) reflection/recreation, (5) transportation, (6) procure and prepare food, and (7) work/school.

The choice of data for this research depends on the fact that time diaries are frequently used in 
time-use research. Statistical bureaus around the world collect diary data in order to study how time is spent in a population. Using real diary data implies a higher complexity in the dataset as different people register their activities with different accuracy and detail. Also, people perform different numbers of activities and in varying order each day. This choice, of real diary data, was however a conscious one as we want to evaluate the representations used for interactively analysing the collected data under real circumstances. The specific data subsets used in the tasks were selected to provide a realistic level of complexity and present appropriate features for the subjects to identify.

\subsection{Visual representation}

In the visual representation used in VISUAL-TimePAcTS the individuals' performed activities, visited places, and companionships are drawn as continuous trajectories in a coordinate system (Figure 2). The $\mathrm{x}$-axis holds the individuals in a population, the $\mathrm{y}$-axis is the time axis and the z-axis can be used to display the activities, places or companionship one at a time. In the remainder of the description we will concentrate on the representation of the activities but the same description also applies for places visited and companionships during an individual's diary day. Each individual's trajectory, the activity or individual path, consists of a tube representing each activity, stretching between the start and end time of this activity, and of lines connecting the separate tubes in order to retain the connectivity during the represented day. Colour is used to represent each of the 7 general activity categories in the data. Black cylinders are used as delimiters at the start of each distinct activity, to highlight the division of the activity categories into the more detailed activity descriptions.

Seen in the front view (Figure 2, left) the activity path resembles a 'stacked' bar-chart revealing the order of occurrence and duration of the activities during the day. Several individuals' activity paths can be drawn beside each other so that the time use of sets of individuals can be compared, forming an 'activity-time cube' (Figure 2, lower row). The activity path seen in the side view (Figure 2, right) reveals the actual path which shows the break up into more detailed descriptions of the performed activities. This view is less meaningful if the number of represented individuals exceeds one, as seen in the lower rightmost part of Figure 2, the individuals' paths overlap making it difficult to distinguish between them. As the number of individuals included in the activity-time cube increases the separation between their paths and the radius of the tubes decreases in proportion to the size of the screen. If the number of individuals gets large enough, however, the activity-time cube begins to resemble a solid block of data, making it impossible to distinguish relationships between paths from any other view apart from the front (2D) one. The number of individuals that can be visually compared in a 3D, free rotation view depends on the screen size, but should probably not exceed 12 on a standard desktop monitor.

\subsection{Visual-TimePAcTS}

The visual analysis tool VISUAL-TimePAcTS consists of a graphical user interface and an interactive visualization window. Complex queries are created dynamically and applied to the activity diary database through the user interface in order to extract subsets of the individuals in the data. Activity paths of the individuals that match the query criteria are then drawn in the visualization window (orthographic projection).

The user can interact with the display freely: rotation, scaling and translation is possible. The user can rotate the displayed objects to examine the representation from different angles, either to align and compare specific data items or to avoid obscuring them. It is also possible to jump from the front to the side view and zoom into or look at the data from a distant point of view. These properties are important when the number of individuals is large and when the activities are drawn with a high level of detail. Mouse clicking on an individual returns detailed information about the individual and the activity performed at the picked time point, which is presented in a separate user interface. A textbox also pops up at the picked point, showing the activity code and description and its start and end time. 
Single activities or ranges of activities can be highlighted in the representation enabling the study of their distribution within the context of the data. Highlighted activities are drawn in colour while the remaining ones are coloured in grey. Furthermore, a semi-automatic sequential mining algorithm is implemented in the tool [4] which extracts all activity sequences meeting some user specified constraints and enables the study of patterns in activity diary data. Finally, VISUAL-TimePAcTS also includes frequency diagrams and simple statistics linking it to traditional analysis methods of time-use data.

\section{Experiment}

The main objective of many studies concerned with identifying patterns in human behaviour, through the use of either spatio-temporal or activity diary data, has been the identification of relationships within and between individuals' trajectories (as pointed out in Section 2). These relationships are, in many cases, retrieved by studying the proximity in space and/or time of the trajectories in 3D time geographical representations: positions in space and time where individuals' trajectories appear to meet or approach. Focusing on activity space and time and studying when, where, and for how long individuals perform activities simultaneously (but not necessarily in company) gives insight to similarities in the way individuals 'build' their days and so the way they live their lives. One may conclude that when 3D time geographical representations are used in the study of spatio-temporal human behaviour a most frequently occurring task is the visual search for similarity (in space or time or both) in trajectories.

The representation of trajectories (activity paths) in VISUAL-TimePAcTS (as described in Section 3) is three dimensional and interactive; allowing a user to rotate and compare the paths from any direction. The typical users of VISUAL-TimePAcTS, however, do not make use of this interaction alternative. They prefer the use of the static 2D representations available for exploring the diary data, these are: the front (Figure 2, left) and side (Figure 2, right) view of the activity-space cube. This brought about doubts as to whether a 3D representation was adding functionality to the tool or whether it was, in fact, superfluous. These doubts raised the questions for this evaluation study.

The visual search for similarities in activity paths in the $2 \mathrm{D}$ front view of the activity-time cube is performed by using the activity category colour as an indicator of activity similarity at a certain time point and retrieving activity details from each individual's path by clicking with the mouse in order to confirm if the individuals indeed perform the same activity. In the static $2 \mathrm{D}$ side view the search for similarities is not possible as the paths overlap completely making it impossible to tell them apart. In a freely rotating activity-time cube performing the same task implies that the user has to identify activities (tubes in the path) having the same activity value (z-axis value) at the same time point (y-axis value). Orientation and depth perception problems of the user with respect to the data complicates the execution of this task. To avoid this problem an alternative interaction scheme has been used where we maintain the side view $\left(90^{\circ}\right.$ rotation) of the activity-time cube as the starting view and constrain the rotation to be around the y-axis (time axis) only while restricting it to 20 degrees towards each direction $\left(70^{\circ}-110^{\circ}\right.$ rotation) (Figure 3$)$. This representation will hereafter constitute the $3 \mathrm{D}$ representation and will be compared to the static front view of the cube which will hereafter be referred to as the $2 \mathrm{D}$ representation.

\section{$4.1 \quad$ Experiment 1}

The purpose of this experiment was to investigate how users carrying out a typical visual exploration task perform in the two representations in VISUAL-TimePAcTS. This typical task involves the user responding to how many activities are concurrently performed by a group of individuals over the time period of one day $(24 \mathrm{~h})$. Concurrently performed is defined as the individuals being engaged in the same activity at the same point in time, although one individual might begin or end the activity earlier or later than the others. We regard this task as typical as it is often the starting point of 


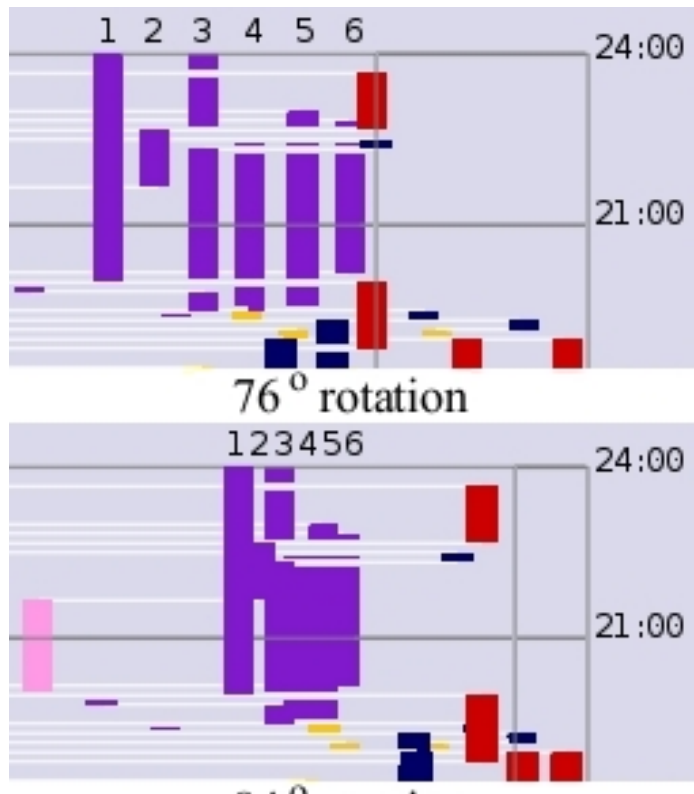

$84^{\circ}$ rotation

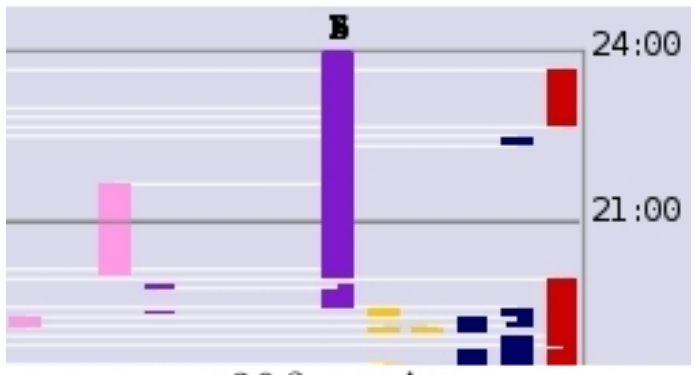

$90^{\circ}$ rotation

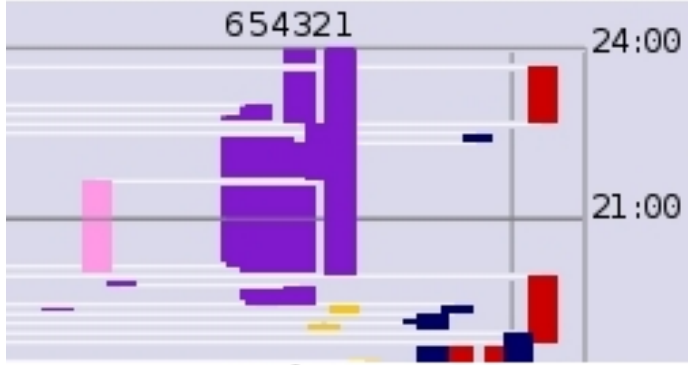

$96^{\circ}$ rotation

$\begin{array}{lllllll}6 & 5 & 4 & 3 & 2 & 1\end{array}$

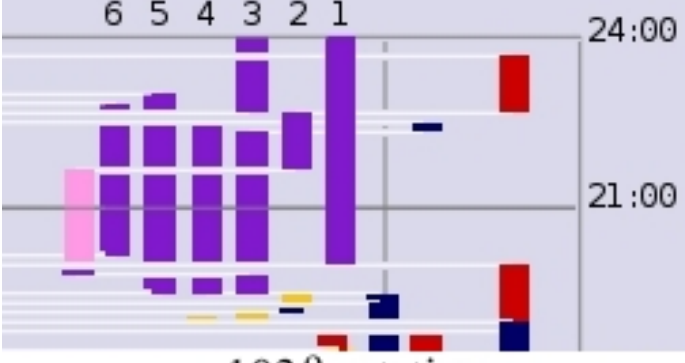

$103^{\circ}$ rotation

Figure 3: Illustration of the constrained rotation of the 3D representation in the VISUAL-TimePAcTS application used in the experiments. Rotation is constrained around the time axis (y-axis) and restricted to $20^{\circ}$. During these slight rotations, bundles of activities are formed at the concurrent activity positions as the activity paths approach $\left(84^{\circ}, 96^{\circ}\right)$ and completely overlap at the initial view state of $90^{\circ}$ (c). 
our users within social science when studying the coexistence of a group of people. Starting from the concurrent activities and studying the activities performed before and after these can lead to insight into the 'helping structures' of a family or the similarity in routines in a larger social group. Further, one and the same activity can be performed concurrently several times during the day since it is not the nature of an activity that is of interest but the number of times that it is concurrently performed.

The motivation for the selected task is that it is both:

- a fundamental question asked by social scientists regarding the complexity of daily life, and hence representative and ecologically valid and

- simple enough to allow a controlled comparison between the two representations yet pinpoint the relevant differences between them.

\subsubsection{Apparatus and Viewing Condition}

The experiment was carried out using a Windows workstation with a Pentium 4 based computer and a Dell 21" LCD monitor set with a resolution of $1280 \times 1024$ pixels. The participants were seated in front of the monitor at a standard viewing distance of approximately $60 \mathrm{~cm}$. A simplified version of the application with reduced functionality was implemented to present the stimuli using the full screen and allow interaction with the program using a mouse and a keyboard. To prevent visual disorientation in the $3 \mathrm{D}$ representation, rotation was restricted to be around the y-axis only (as described in Section 4 and shown in Figure 3).

\subsubsection{Stimuli and Task}

Each stimulus displays activity paths of the individuals in a family over the time period of one day (24h). The representation described in section 3.2 was used. A family was comprised of either two or four individuals. An experimental assessment like this requires clearly defined answers [25] therefore, a controlled set of stimuli constructed from a selection of sixteen families out of the activity diary data set was used. Each family was displayed once in the 2D trial and once in the 3D trial resulting in thirty-two distinct stimuli $(16 \times 2)$. An example of the same 4 individual family type, as seen in the 2D and 3D trial, is shown in Figure 4. The number of activities concurrently performed by the individuals in each stimulus was constrained to be between zero and three and there were two stimuli within each family type having the same number of concurrent activities $(4 \times 2 \times 2)$. In order to minimize any use of response strategies, however, the participants were not informed about any of these facts, apart from that the answer could be none or several concurrent activities.

The task in any individual trial was to examine the displayed data and respond stating the total number of occasions where all members of the family shown were engaged in the same activity at the same time. Using the $2 \mathrm{D}$ representation this involves a systematic interaction process during which memorization of the displayed data is necessary. First, each bar representing one individual has to be compared with the others to locate activities having the same colour (meaning belonging to the same activity category) at the same interval in time (position). However, colour does not tell what specific activity is being performed, only the general category to which it belongs. Therefore, the next step is to click on the activities in order to get details about them in the pop-up text box (as seen in Figure 4). If the performed activity is the same for all displayed individuals then it should be added (remembered) to the number concurrently performed and the search continues until all possible concurrencies have been examined. The pop-up text box also gives details about the activities start and end times which may be needed when these times are very close to each other making comparison between bars difficult. As an example, if one individual ends an activity at 11:00am and another begins the same activity at 11:05am it may be difficult to see whether they are performed concurrently by visual means alone. 


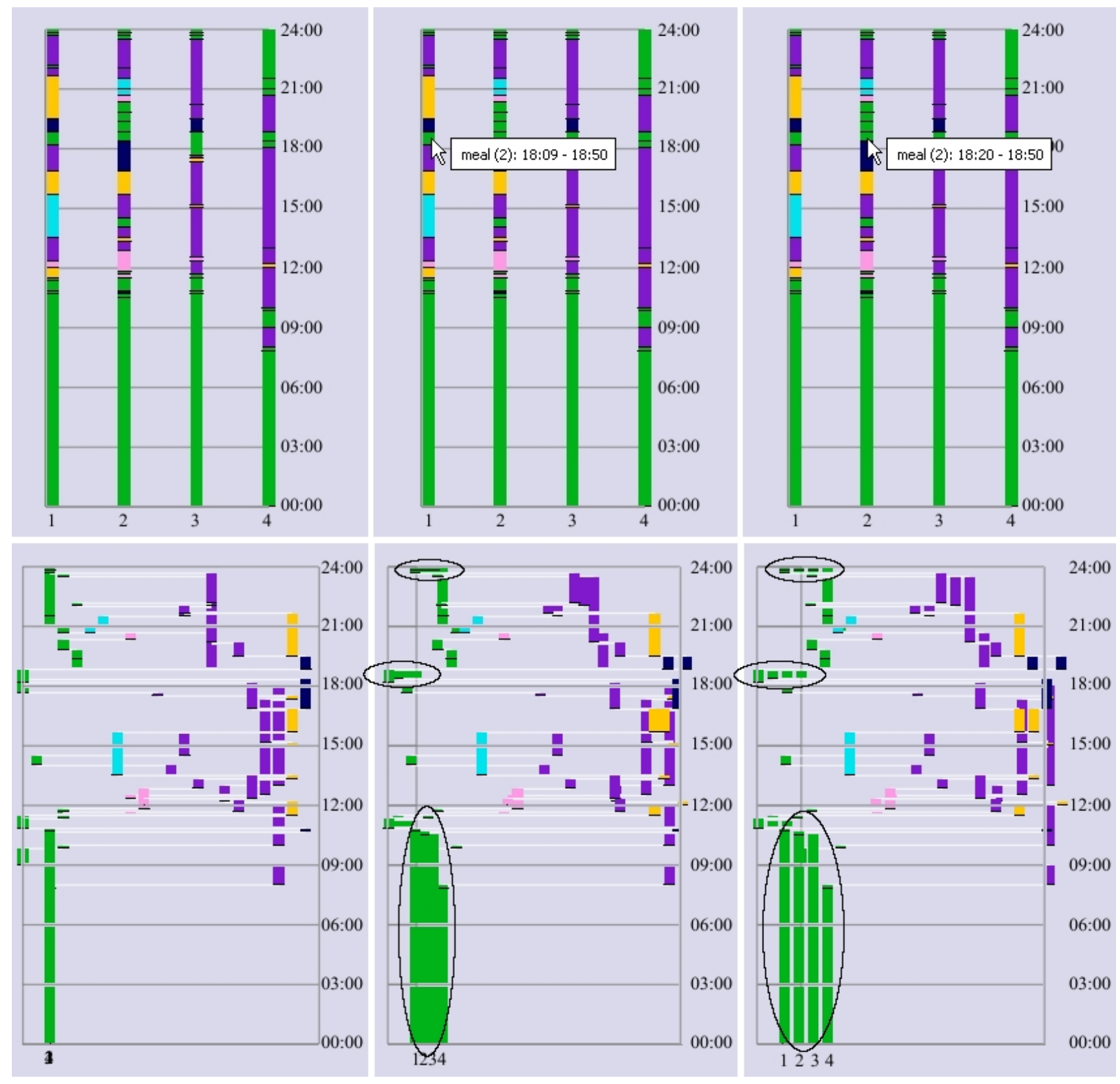

Figure 4: Example stimulus display of a 4 individuals family type. Top row: stimulus in the 2D trial. Bottom row: $3 \mathrm{D}$ trial with $90^{\circ}, 95^{\circ}$ and $100^{\circ}$ rotation respectively. In $2 \mathrm{D}$ the task is performed through clicking on the activity paths and in $3 \mathrm{D}$ through rotating the view to reveal clusters of activities.

Using the 3D representation the task can be solved in a more straightforward way requiring less memorization and simpler interaction. In this side view the categories are displayed along the activity axis in the activity path, hence displaying all separate activities and not only the general activity categories. To search for concurrent activities the representation is rotated slightly back and forth (constrained to 20 degrees in each direction) as compared with the clicking in the static 2D front view. When an activity of one individual overlaps an activity of another individual at the intitial rotation position (90 degrees), then this means that they are performed concurrently. This rotation and identification process is shown in Figure 3. One can see that during the slight rotation the activities form bundles as they approach, indicating that they are in fact concurrent. The fact that the rotation is restricted to be around the y-axis makes concurrent activities relatively easy to spot (Figure 4). Hence, in this representation colour and position provide enough information to solve the 
task, the user does not need to memorize any activity names, and the interaction requires less clicking for details.

\subsubsection{Experimental Design}

The study was designed as a three-factor mixed factorial design with all factors having two levels each. There was one within-subject factor: task type (family with two individuals vs. family with four). The between-subject factors were representation method (2D vs. 3D) and order of presentation of task type (begin trial with two individuals vs. begin with four). This means that all participants were shown the same stimuli in different representations. Each participant performed both types of task (two and four individuals) using one of the representations (2D or $3 \mathrm{D})$ ), completing all trials in the first task type before beginning the second task type. The rationale for not having the same person using both types of representation is that it avoids any transfer effects biasing the result (such as learning the data set). Balancing the order of presentation of tasks also provides an opportunity to prevent the ordering from having an effect since, for example, practice or boredom will be equalized out. Participants were randomly assigned to each of the two representations and order of presentation. Each task type consisted of eight trials and the four possible numbers of concurrent activities occurred twice each in a randomized order of presentation. To summarize, each participant viewed a total of sixteen different stimuli.

\subsubsection{Procedure}

The experiment was performed in two parts: one introductory training part followed by a timed trial part. The participants first signed an informed consent and filled in a questionnaire asking for demographic information and computer experience, in particular their experience with information visualization tools. Each participant then reviewed writen instruction materials explaining the task, the procedure and how to interact with the computer program. Participants were instructed to be as accurate as possible. They were also informed that they were being timed and that answers could not be changed. Thereafter, a session with eight training trials (four trials of each task type) followed (not being timed). Here questions were allowed and each answer was confirmed with feedback from the experimenter so that participants were consistently trained before the timed trials part began. The sequence of trials was self-paced and the participants controlled the screen by pressing the space bar when they wished a trial to begin. Each trial consisted of a sequence of four images. First a blank image was presented. Pressing the space bar caused the first stimulus to appear, which remained until space was pressed once again. An image then appeared consisting of a dialog box where the participant typed their answer: the correct result being a figure between zero and three. Clicking on an OK button then caused the blank display to reappear and this finished the trial. The procedure was repeated for each trial. For each trial, error and response time were recorded (response time did not include time for entering the subjects answer). No feedback was provided. An experimental session lasted, at most, 40 minutes (including the introductory and training part).

\subsubsection{Participants}

A total of 20 persons took part in the experiment. They were undergraduate students at the University Campus and recruited from student populations of occupational therapy (11), teaching (7) and social science (2). All of the participants declared that they had normal, or corrected to normal, vision and normal colour vision. Their age varied between 20 and 41 years with a median age of 22 . None of the participants stated that they were familiar with the kind of time-use visualization used in the experiment and they had no prior knowledge about the purpose of the test. The participants received a small compensation for taking part in the experiment. 


\subsection{Results}

Data from all twenty participants were analysed. For all statistical tests, $\alpha$ levels of significance were $p<0.05$. A summary of descriptive statistics is shown in Table 1.

The distributions of the errors deviated significantly from the normal (by means of ShapiroWilk test and the Kolmogorov-Smirnov test) subsequent tests were, therefore, performed using nonparametric methods (Mann-Whitney U and Wilcoxon signed-rank test). First, the number of errors for the different main conditions was tested. The $2 \mathrm{D}$ representation produced more errors, the median value $(\mathrm{Mdn})$ being 3 , than the $3 \mathrm{D}$ representation $(\mathrm{Mdn}=1.5)$ and the difference was significant $(U=23.50, p=0.042, Z=-2.055, N=20)$. When the error data were collapsed over representation methods analyzing a difference between tasks the task having two individuals was found to produce fewer errors $(\mathrm{Mdn}=0.5)$ than the one having four $(\mathrm{Mdn}=1.5)$ but the difference was not significant $(T=43.5, Z=-1.608, p=0.062)$. No significant effect of order of presentation of tasks was found $(U=38.5, p=0.388, Z=-0.892, N=20)$. Next, the difference in errors between the two task types for each representation method separately, was examined. In 2D there was no significant difference between task types $(T=17, Z=-0.142, p=0.496)$. For the 3D representation, however, the difference between task types was significant $(T=3.5, Z=-2.332, p=0.027)$ : the four individuals task type produced more errors $(M d n=1.5)$ than the two individuals one $(M d n=0)$. Finally, the difference between task types was examined for both representations. For the task having two individuals there was a significant difference $(U=24.00, p=0.045, Z=-2.136, N=20)$, the $2 \mathrm{D}$ representation produced more errors $(\mathrm{Mdn}=1.5)$ than the $3 \mathrm{D}$ representation $(\mathrm{Mdn}=0)$. However, in the task type having four individuals no significant difference $(U=45.00, p=0.807, Z=-0.386, N=20)$ was found between $2 \mathrm{D}(\mathrm{Mdn}=1.5)$ and $3 \mathrm{D}(\mathrm{Mdn}=1.5)$.

The response time data was analysed with a mixed ANOVA (Analysis of Variance). There was a significant main effect of representation method $(F(1,16)=43.403, p<0.001)$. The response times were more than twice as long for the 2D representation (mean 53.9 seconds) compared to the 3D representation (mean 19.88 seconds). There was also a significant main effect of task type $(F(1,16)=168.894, p<0.001)$. The mean value $(48.34$ seconds $)$ for the task having four individuals was almost twice as high as the mean value for the task having two (25.45 seconds). No significant effect of order was found $(F(1,16)=43.408, p=0.821)$. Finally, a significant effect of interaction was present $(F(1,16)=31.459, p<0.001)$. When the number of individuals increased from two to four there was a greater increase in difference in response time when using the $2 \mathrm{D}$ representation than when using the 3D one. The difference between 2D and 3D for the task type having two individuals is 24.19 seconds and 43.9 seconds for the one having four individuals.

\begin{tabular}{l|cccccc|cc} 
& $\begin{array}{c}\text { Nr } \\
\text { trials }\end{array}$ & $\begin{array}{c}\text { Total } \\
\text { errors }\end{array}$ & Max & Median & $\begin{array}{c}\text { Mean } \\
\text { errors }\end{array}$ & $\begin{array}{c}\text { Std } \\
\text { time }\end{array}$ & $\begin{array}{c}\text { Mean } \\
\text { time }\end{array}$ & Std \\
\hline \hline $\mathbf{2 D}$ & 160 & 31 & 4 & 2.0 & 1.55 & 1.099 & 53.91 & 22.27 \\
$\mathbf{3 D}$ & 160 & 18 & 3 & 1.0 & 0.9 & 0.968 & 19.88 & 9.62 \\
\hline $\mathbf{2} \mathbf{i}$ & 160 & 19 & 4 & 0.5 & 0.95 & 1.146 & 25.45 & 15.28 \\
$\mathbf{4} \mathbf{i}$ & 160 & 30 & 3 & 1.5 & 1.5 & 0.946 & 48.34 & 26.25 \\
\hline $\mathbf{2 D} / \mathbf{2} \mathbf{i}$ & 80 & 15 & 4 & 2.0 & 1.5 & 1.269 & 37.53 & 11.90 \\
$\mathbf{3 D} \mathbf{2} \mathbf{i}$ & 80 & 4 & 2 & 0.0 & 0.4 & 0.699 & 13.38 & 5.24 \\
\hline $\mathbf{2 D} / \mathbf{4} \mathbf{i}$ & 80 & 16 & 3 & 1.5 & 1.6 & 0.966 & 70.29 & 17.60 \\
$\mathbf{3 D} / \mathbf{4} \mathbf{i}$ & 80 & 14 & 3 & 1.5 & 1.4 & 0.966 & 26.39 & 8.6
\end{tabular}

Table 1: Descriptive statistics from experiment 1 ( $\mathrm{i}=$ individuals). 


\subsection{Discussion of Results}

To summarize the results, the $2 \mathrm{D}$ representation produces more errors and requires longer time to use compared to the 3D representation. There is a difference between tasks such that a comparison of four individuals produces more errors and takes longer time than a comparison of two individuals. When using the $2 \mathrm{D}$ representation the amount of errors is about the same regardless of the number of individuals compared. Hence, solving the task of determining the amount of concurrent activities in terms of errors is as difficult when there are two as when there are four individuals. In the 3D representation however, the number of errors increases when the level of complexity (more individuals) increases. This was not expected and undermines the use of 3D for tasks of this kind. Concerning response times, when the number of individuals increases from two to four there is a greater increase in difference in response time when using the $2 \mathrm{D}$ representation than when using the $3 \mathrm{D}$ one.

To conclude, when task complexity increases from two to four individuals so do search times. However, the fact that the amount of error also increased in the 3D representation was a surprise. Therefore it seemed necessary to further investigate this trend with a new experiment. If the performance continues to decrease with complexity, then this may indicate that the $3 \mathrm{D}$ representation is not well suited for this kind of data exploration.

\subsection{Experiment 2}

Using the same basic paradigm as in the first experiment this second experiment aimed to further study the use of the 3D representation. The purpose was to investigate if, and then how, an increase in the number of individuals displayed would affect task performance. An answer to this question will help in deciding on the usefulness of the representation.

\subsection{Stimuli and Task}

The basic task and stimulus used were of the same type as adopted in the 3D condition in experiment 1. However, in this second experiment the number of individuals in the displayed groups (the level of task type) was now two, four, six and eight respectively. Activity diary data from twenty-four new groups of individuals were selected from the diary data set.

\subsection{Participants}

Twelve new participants aged between 21 and 35 (median age was 22) took part in this second experiment. They were all undergraduate students at the University Campus and recruited from student populations of occupational therapy (4), teaching (3) and social science (5). All stated that they had normal colour vision and normal, or corrected to normal, vision. None of the participants had any prior experience of time-use visualization software and they were all unaware of the purpose of the experiment. The participants received a small compensation for taking part.

\subsection{Experimental Design}

The experiment was designed as a within-subjects design with one factor: task type having four levels (two, four, six and eight individuals). The order of presentation of the four levels was randomly assigned to each participant. Each task type consisted of six trials for each level (this design yielded a total of $24(4 \times 6)$ trials per participant). The number of possible concurrent activities again ranged between 0 and 3. Each possibility appeared once within each level of task type and for the remaining two trials the number was randomly selected. The rationale for this was to avoid the possibility of participants acquiring a response strategy and hence biasing the results. In all other respects the method used was identical to that used in experiment 1 . An experimental session lasted approximately 30 minutes. 


\subsection{Results}

Data from all twelve participants were analysed and, again, $\alpha$ levels of significance were $p<0.05$. The descriptive statistics are presented in Table 2.

The error data deviated significantly from the normal (Kolmogorov-Smirnov and Shapiro-Wilk) and so the nonparametric test Friedman ANOVA by ranks was used for further analysis. The result showed no significant difference in terms of errors between the different levels of task (Chi Sqr. $(N=$ $12, d f=3)=7.167, p=0.062$ ). It is, however, close to the 0.05 level.

The response times were analyzed by means of a repeated measures analysis of variance (ANOVA). Before the test the data was transformed using a natural logarithm since this improved the fit to a normal curve. The results showed a significant difference between the four levels of complexity $(F(3,33)=14.672, p<0.001)$. According to the follow up Bonferroni-corrected pairwise comparisons, the task having two individuals differed from the other three with all probabilities below $p<0.01$. No other statistically significant differences were found.

\begin{tabular}{c|cccccc|cc} 
& $\begin{array}{c}\text { Nr } \\
\text { trials }\end{array}$ & $\begin{array}{c}\text { Total } \\
\text { errors }\end{array}$ & Max & Median & $\begin{array}{c}\text { Mean } \\
\text { errors }\end{array}$ & $\begin{array}{c}\text { Std } \\
\text { time }\end{array}$ & $\begin{array}{c}\text { Mean } \\
\text { time }\end{array}$ & Std \\
\hline \hline $\mathbf{2} \mathbf{i}$ & 72 & 7 & 2 & 0 & 0.58 & 0.79 & 16.96 & 3.26 \\
$\mathbf{4} \mathbf{~ i}$ & 72 & 12 & 2 & 1 & 1 & 0.74 & 28.10 & 8.09 \\
$\mathbf{6} \mathbf{~ i}$ & 72 & 7 & 2 & 0.5 & 0.58 & 0.70 & 29.31 & 7.37 \\
$\mathbf{8} \mathbf{~ i}$ & 72 & 3 & 1 & 0 & 0.25 & 0.45 & 27.31 & 11.74
\end{tabular}

Table 2: Table 2: Descriptive statistics from experiment 2 (i=individuals).

\subsection{Discussion of Results}

This experiment aimed at investigating if, and then how, an increase in task complexity would affect performance when using the $3 \mathrm{D}$ representation. The results show that, in terms of search times, there is a difference between the low complexity level (two individuals) and the other three more complex ones, however among these three performances are comparable. Regarding errors no significant differences were found, although, the highest number of errors was, once again, found in the complexity level having four persons. This task complexity seemed to be more difficult than the others and according to typical comments by participants the task got easier when the number of individuals increased. This is, probably, best explained by the visually distinct bundles that the increase in the number of individuals creates and how the representation can be improved to better emphasize these structures will be further discussed in the next section. Hence, we can conclude that performance does not continue to deteriorate as data complexity increases. The results indicate that the $3 \mathrm{D}$ representation can indeed also be used for analyzing larger groups of people.

\section{General Discussion and Conclusions}

The experiments in this study have answered two main research questions. The first experiment was performed in order to establish whether there was a difference between the $2 \mathrm{D}$ and the $3 \mathrm{D}$ time geographical representations currently used for analysing activity diary data in VISUAL-TimePAcTS in supporting users performing a representative task: identifying when a group of individuals perform activities concurrently. Overall, the outcome was in favour of the 3D representation but in the 3D the error rate increased substantially with task complexity. This raised the question examined in the second experiment, which was to determine whether this trend would continue and performance would gradually deteriorate with increasing task complexity or whether performance would remain stable across increasing group sizes. According to the results the breaking point seems to be above the simplest level of including two persons and thereafter performance is comparable up to groups of eight 
individuals. In fact, the 'bundles' (clusters) constructed by the concurrent activities become larger and more distinguishable as the number of individuals (task complexity) increases. Hence, summing up the results, we may conclude that the $3 \mathrm{D}$ representation is in fact appropriate for performing the identified class of representative task. Further, it is superior to the $2 \mathrm{D}$ representation both in terms of speed and accuracy. It also seems fair to predict that when task complexity increases, the 2D representation will ultimately become overwhelming in terms of memory load and number of interactions required.

An issue that needs mentioning, however, is the performance 'dip' for the four individuals case in the 3D representation, which produces more errors in both experiments. We suspect that four is the turning point for the formation of bundles of paths in the type of diary data that we have considered. For reasons explained in section 3.1 we have chosen to use real instead of simplified synthetic activity diaries. This has implied more fragmentation in the dataset leading, in turn, to a possible increase of clutter. Comparing two individuals' paths is relatively easy as there is not enough data on the screen to make it confusing. Considering the experimental results, comparison of six or more benefits from the formation of bundles as previously described. This leads us to suspect that comparison of four individuals is left in the middle including enough individuals to make the representation confusingly cluttered but not enough to create distinct clusters of activities and thus creating the peak in the error rate.

There are further issues that arise regarding both representations that need to be discussed. This will be done in the remainder of this section and some suggestions for overcoming these issues will be presented. These suggestions have been implemented but currently remain untested.

The first issue we review concerns separation. In the $2 \mathrm{D}$ representation a larger number of individuals can be compared with each other without incurring the cluttering problems of 3D. The comparison in 2D (as described in section 4.1.2) is dependent on the spacing between the individuals. One can more easily determine whether two activities occur at the same time if the paths are adjacent particularly when the activities' start and end times are very close. The separation between the activity paths is also significant in the $3 \mathrm{D}$ representation since it affects the ability of the user to perceive the bundles of paths, through the rotation around the y-axis (Figure 3). If they are drawn near to one another the paths appear to move together during rotation. If they are drawn too close, however, and the number of individuals on display is large there might be interference between the paths leading to the identification of 'false' bundles. For example activities that are close to one another on the activity axis, might be hard to distinguish between; or it may be difficult to tell if all individuals are actually performing the activity under investigation. Further experimentation is needed in order to decide whether there is an optimal separation distance or ratio that applies to all views, and what that might be. Until this future work is performed we have implemented a dynamic slider that allows the user to change the spacing between the paths depending on the view and the number of individuals under study.

The combination of separation and rotation leads us to the next issue which is the style of rotation, itself, in the 3D representation. In the experiments rotation was performed around a central $y$-axis. This has the effect that the apparent displacement of the activities due to the rotation applied increases with an increased distance along the $\mathrm{x}$-axis from the rotation axis. This leads to problems of increased cluttering, especially at larger rotations, since the data becomes more clumped towards the centre along the screen's $\mathrm{x}$ direction. By replacing the rotation with a shearing transformation, such that the activities are translated in proportion to their distance along the z-axis from the origin (the central y-axis)(see Figure 5), the displacement of activities is uniform over the whole display resulting in the dual benefits that cluttering is reduced and that the bundles are revealed with small rotations, uniformly over the display.

Depth perception in the $3 \mathrm{D}$ representation is also an issue we have addressed. In the initial representation apart from 3D interaction no other depth cues were present making it hard to distinguish which activities (tubes) belong to the same path as well as which are in front of others. Applying self shadowing to the activity paths has improved the ability to discriminate between the paths and 


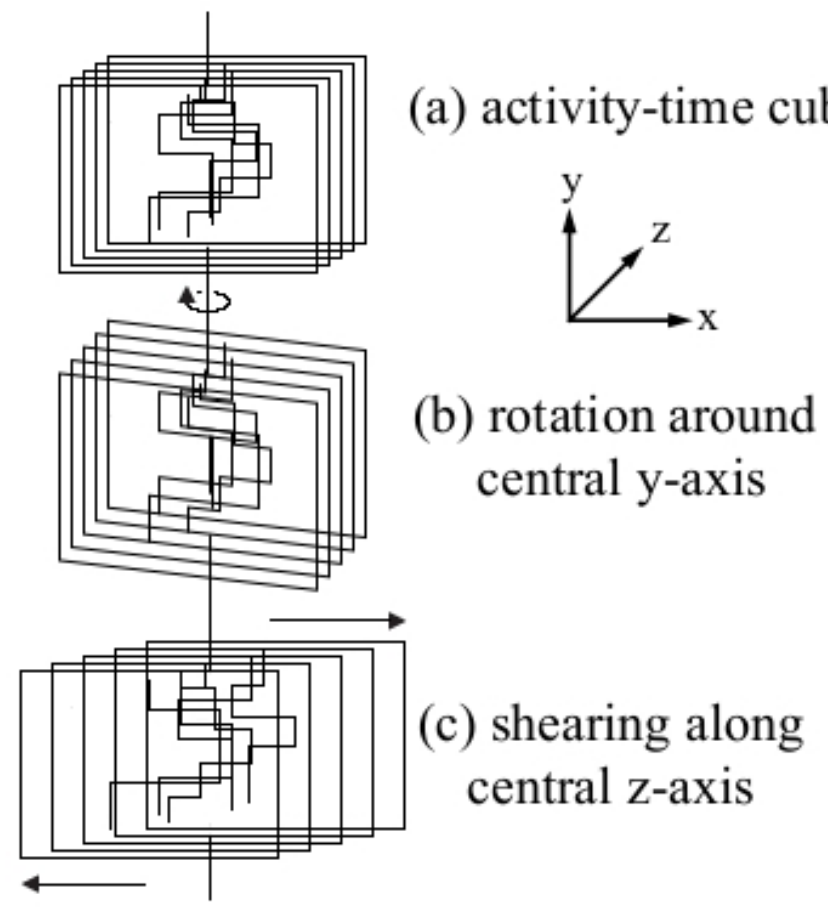

Figure 5: Illustration of shearing of the activity-time cube along the central z-axis as compared to rotating around the central y-axis.

implementing aerial perspective in the scene has further enhanced the depth perception.

The issues discussed this far might be thought specific to the representation available in the visual analysis tool VISUAL-TimePAcTS, which employs a more simplified time geographical representation that does not consider geographical space. Having established, however, that one of the most frequently performed tasks for time geographical representations is the visual search for relationships, identified as bundles in the data whether examining activity-time or space-time paths, some of the previously discussed issues can be generalized. The constrained rotation is an example of such an issue. Free rotation in a time cube can in many cases be more confusing than helpful when trying to identify groupings in time in the data. In fact, the geographical map in space-time cubes is seldom directly used. It is, of course, important since it provides the geographical reference but the exploration process is most often primarily focused on the trajectories which evolve over the map. Searching for relationships between these trajectories involves identifying times when they meet, or identifying where people are at the same time and what they do. A rotation constrained around the time axis may, in this respect, decrease orientation problems and make the relationships easier to spot. Having been identified, they could then be studied through another interaction scheme. Furthermore, the number of paths that can be studied simultaneously and efficiently in a time cube is limited. We have in this paper shown that such a representation works efficiently for at least 8 people, but we suspect that when the number of compared individuals' paths increases over a certain limit the ability to distinguish detail in the paths will decrease. Rotation around the time axis, however, will still provide indications of bundles among paths and these too could then be extracted and examined in another representation. The conclusion that can be drawn here then is that the time cube with constrained rotation is a well suited representation for feature detection in individuals' trajectories. It can be used as an identification and selection view and be combined with other coordinated views where the selected individuals can be studied further.

To conclude, in this paper we have identified tasks commonly performed in the analysis of time geographical data using a time cube. We have related these tasks to those of the study of activity diaries in a time geographical representation. We have then evaluated the performance of the tasks in 
$2 \mathrm{D}$ and 3D representations currently used for analysis of activity diaries and have demonstrated that a 3D representation with a rotation constrained around a single axis is advantageous in the visual search for relationships.

\section{References}

[1] Hägerstrand T. What about people in regional science? Papers in Regional Science 1970; 24(1): 6-21.

[2] Kapler T, Wright W. GeoTime information visualization. IEEE Symposium on Information Visualization 2004 (Austin, Texas, USA, 2004), IEEE Computer Society: Washington, DC; 25-32.

[3] Ellegård K. A time-geographical approach to the study of everyday life of individuals - a challenge of complexity. GeoJournal 1999; 48(3): 167-175.

[4] Vrotsou K, Ellegård K, Cooper M. Everyday life discoveries: mining and visualizing activity patterns in social science diary data. 11th International Conference Information Visualisation 2007, IV'07 (Zürich, Switzerland, 2007), IEEE Computer Society; 130-138.

[5] Lenntorp B. Paths in space-time environments: a time-geographic study of movement possibilities of individuals. Royal University of Lund, Department of Geography; 1976, 150 pp.

[6] Peuquet DJ. It's about time: a conceptual framework for the representation of temporal dynamics in geographic information systems. Annals of the Association of American Geographers 1994; 84(3): 441-461.

[7] Ellegård K, Hägerstrand T, Lenntorp B. Activity organization and the genration of daily travel: two future alternatives. Economic Geography 1977; 53(2): 126-152.

[8] Lenntorp B. The drama of real-life in a time-geographic disguise. Sixth Theo Quant Meeting 2003 (Besancon, France, 2003).

[9] Forer P. Geometric approaches to the nexus of time, space and microprocess: implementing a practical model for mundane socio-spatial systems. In: Egenhofer MJ, Golledge RG (Eds). Spatial and Temporal Reasoning in Geographic Information Systems. Oxford University Press, New York; 1998. 171-190.

[10] Dykes JA, Mountain DM. Seeking structure in records of spatio-temporal behaviour: visualization issues, efforts and applications. Computational Statistics \& Data Analysis 2003; 43(4): 581-603.

[11] Kwan MP. Interactive geovisualization of activity-travel patterns using three-dimensional geographical information systems: a methodological exploration with a large data set. Transportation Research Part C: Emerging Technologies 2000; 8: 185-203.

[12] Zhao J, Forer P, Harvey AS. Activities, ringmaps and geovisualization of large human movement fields. Information Visualization 2008; 7(3-4): 198-209.

[13] Miller HJ. What about people in geographic information science? Computers, Environment and Urban Systems 2003; 27(5): 447-453.

[14] Kraak MJ. The space-time cube revisited from a geovisualization perspective. 21st International Cartographic Conference 2003 (Durban, South Africa, 2003); 1988-1995.

[15] Yu H. Spatio-temporal GIS design for exploring interactions of human activities. Cartography and Geographic Information Science 2006; 33(1): 3-19. 
[16] Kwan MP. Gender, the home-work link, and space-time patterns of nonemployment activities. Economic Geography 1999; 75(4): 370-394.

[17] Kwan MP, Lee J. Geovisualization of human activity patterns using 3D GIS: A time-geographic approach. In: Goodchild MF, Janelle DG (Eds). Spatially integrated social science. Oxford University Press, USA; 2004. 48-66.

[18] Kwan MP. GIS methods in time-geographic research: geocomputation and geovisualization of human activity patterns. Geografiska Annaler: Series B, Human Geography 2004; 86(4): 267280 .

[19] Gatalsky P, Andrienko N, Andrienko G. Interactive analysis of event data using space-time cube. 8th International Conference Information Visualisation 2004, IV'04 (London, UK, 2004), IEEE Computer Society; 145-152.

[20] Turdukulov UD, Kraak M, Blok CA. Designing a visual environment for exploration of time series of remote sensing data: In search for convective clouds. Computers \& Graphics 2007; 31(3): 370379.

[21] Kristensson PO, Dahlbäck N, Anundi D, Björnstad M, Gillberg H, Haraldsson J, Mårtensson I, Nordvall M, Ståhl J. The trade-offs with space time cube representation of spatiotemporal patterns. Available at http://www.citebase.org/abstract?id=oai:arXiv.org:0707.1618. 2007.

[22] Ellegård K, Cooper M. Complexity in daily life - 3D-visualization showing activity patterns in their contexts. electronic International Journal of Time Use Research (eIJTUR) 2004; 1: 37-59.

[23] Ellegård K, Vrotsou K. Capturing patterns of everyday life - presentation of the visualization method VISUAL-TimePAcTS. 28th IATUR Conference 2006 (Copenhagen, Denmark, 2006).

[24] Vrotsou K, Ellegård K, Cooper M. Interactive visual exploration of time-use data. Poster in 10th International Conference Information Visualisation 2006, IV'06 (London, UK, 2006).

[25] Keim DA, Bergeron D, Pickett R. Test data sets for evaluating data visualization techniques. In: Grinstein G, Levkowitz H (Eds). Perceptual Issues in Visualization. Springer-Verlag, Berlin; 1995. 9-22. 